\title{
CONCEPTUAL DESIGN STUDY OF HORN POWER SUPPLY *
}

\author{
W. Zhang ${ }^{\xi}$, J. Sandberg, W-T Weng \\ Brookhaven National Laboratory \\ Upton, NY 11973
}

\begin{abstract}
A $250 \mathrm{kA}$ pulsed power supply is required for the focusing horn of the proposed Brookhaven AGS Super Neutrino Beam Facility for long baseline neutrino oscillation experiment. It is expected to pulse at $2.5 \mathrm{~Hz}$ repetition rate. A preliminary study is being conducted to explore the key issues associated with the power supply system design. Advanced technologies used in similar systems as well as new ideas are being examined, simulated and evaluated. This power supply will be a very high stored energy, high average power, and high peak power system.
\end{abstract}

\section{INTRODUCTION}

The newly proposed Brookhaven AGS Super Neutrino Beam Facility will be the proton driver for the Very Long Baseline Neutrino Program [1]. A half-megaton water Cerenkov detector will be located deep underground in the Homestake Mine at Lead, South Dakota. The Homestake has been selected as the future site of the Deep Underground Science and Engineering Laboratory (DUSEL) by National Science Foundation recently.

The physics goal of the program is to observe the oscillation pattern as a function of neutrino beam energy, covering three full oscillations yielding precise resolution of all interested parameters. A unique aspect of the BNL proposal is the ability to measure CP parameters with $v_{u}$ beam alone [3].

Two critical factors [2] for the success of the program are the very long baseline (over $2500 \mathrm{~km}$ ) and the specific few-GeV energy band of neutrino beam. The distance from Brookhaven National Laboratory to the Homestake Mine is about 2540 kilo-meters, as shown in Figure 1. An upgraded AGS will provide a IMW "Super Neutrino Beam" covering $1.0 \mathrm{GeV}$ to $7.0 \mathrm{GeV}$ wideband. The main facility upgrade will include a new addition of $1.2 \mathrm{GeV}$ Super-conducting LINAC, a higher repetition rate of 2.5 $\mathrm{Hz}$ in AGS, and a $1.0 \mathrm{MW}$ target station and neutrino channel.

A target and the beam focusing horn system are essential components of the accelerator based neutrino facility. The $28 \mathrm{GeV}$ proton beam from AGS will be transported to a graphite based carbon-carbon composite target, and large number of pions will be generated and decay into muons and neutrinos. A pulsed horn is required to focus the pion beam.

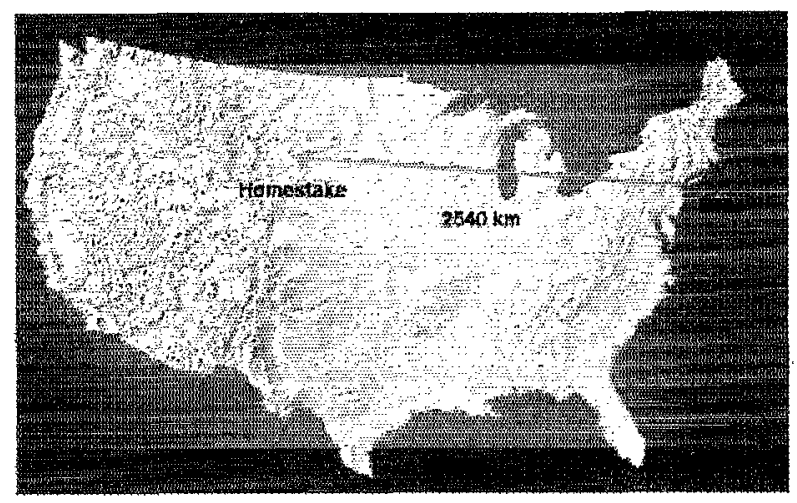

Figure 1. BNL $\rightarrow$ Homestake Super Neutrino Beam.

\section{BASIC SYSTEM DESCRIPTION}

The main objectives of the horn power supply design is to achieve an output pulse amplitude of $250 \mathrm{KA}$, a pulse flat top of $2.6 \mu \mathrm{s}$ or longer with a $1 \%$ flatness and pulse to pulse repeatability, and a repetition rate of 2.5 pulses per second. The main parameter specification is listed in Table 1.

Table 1. Horn/Target design parameters.

\begin{tabular}{|l|l|}
\hline Proton Beam Energy & $28 \mathrm{GeV}$ \\
\hline Protons per Pulse & $8.9 \times 10^{13}$ \\
\hline Average Beam Current & $35.7 \mu \mathrm{A}$ \\
\hline Repetition Rate & $2.5 \mathrm{~Hz}$ \\
\hline Pulse Length & $2.58 \mu \mathrm{s}$ \\
\hline Number of Bunches & 23 \\
\hline Number of Protons per Bunch & $3.87 \times 10^{12}$ \\
\hline AGS Circumference & $807.1 \mathrm{~m}$ \\
\hline Bunch Length & $40 \mathrm{~ns}$ \\
\hline Bunch Spacing & $60 \mathrm{~ns}$ \\
\hline Normalized Emittance - X & $100 \pi$ mm-mrad \\
\hline Normalized Emittance - Y & $100 \pi$ mm-mrad \\
\hline Longitudinal Emittance & $5.0 \mathrm{eV}$-sec \\
\hline Target Material & carbon-carbon \\
& composite \\
\hline Target Diameter & $1.2 \mathrm{~cm}$ \\
\hline Target Length & $80 \mathrm{~cm}$ \\
\hline Hom Small Radius & $7 \mathrm{~mm}$ \\
\hline
\end{tabular}

\footnotetext{
* Work performed under Contract Number DE-AC02-98CH10886 with the auspices of the US Department of Energy.

ร email: arling@bnl.gov
} 


\begin{tabular}{|l|l|}
\hline Beam Size (Radius) on Target & $2 \mathrm{~mm}$ (rms) \\
\hline Horn Smallest Radius & $6 \mathrm{~mm}$ \\
\hline Hom Large Radius & $61 \mathrm{~mm}$ \\
\hline Hom Inner Conductor Thickness & $2.5 \mathrm{~mm}$ \\
\hline Horn Minimum Thickness & $1 \mathrm{~mm}$ \\
\hline Horn Length & $217 \mathrm{~mm}$ \\
\hline Horn Peak Current & $250 \mathrm{kA}$ \\
\hline Current Repetition Rate & $2.5 \mathrm{~Hz}$ \\
\hline Power Supply Waveform & $\begin{array}{l}\text { Sinusoidal, Base } \\
\text { Width } 1.2 \mathrm{~ms}\end{array}$ \\
\hline
\end{tabular}

Most commonly used scheme is the capacitor discharge type. In this type of circuit, a capacitor bank stores the energy, and a main discharge switch release the energy to the load through transmission lines. For very long distant transmission, pulsed transformers have been added into the KEK design and CNGS horn system. The horn as an electrical load is usually being described as an inductor in series with a resistor. Discrete parameters of inductance and resistance are also used to formulate the short length, low impedance transmission lines when associated with low bandwidth pulse. Hence, the circuit can be simplified as a RLC discharge circuit.

The pulse rise time, $T_{r}$, is usually approximated by the quarter period of sine wave. For a lossless LC oscillation circuit, this can be determined by the equation $T_{r}=(\pi / 2) \sqrt{L C}$, and the load current is given by $I(t)=V_{a} \sqrt{C / L} \sin (t / \sqrt{L C})$. The maximum current amplitude is $I_{\max }=V_{o} \sqrt{L / C}$ for lossless LC circuit.

In the case of critical damped RLC cireuit, $R=2 \sqrt{L / C}$ and $I(t)=\left(V_{o} / L\right) t e^{-R t / 2 L}$. The current rise time is $T_{r}=2 L / R=\sqrt{L C}$, and the maximum output current is given by $I_{\max }(t=2 L / R)=V_{\theta} \sqrt{C / L} e^{-1}$.

Compare two cases, for a given $\mathrm{L}, \mathrm{C}$ and charging voltage $V_{0}$, the output current of the critical damped system has peak amplitude of $36.79 \%$ of the lossless one. To reach the same maximum output current, the initial voltage of the critical damped circuit has to be 2.7183 times of the lossless one. It implies that, for the critical damped one, the energy stored in the capacitor bank as well as the charging power has to be 7.389 times larger.

In the high current path, the resistance caused voltage drop and energy dissipation are critical factors to be considered. The cooling system for heat removal from the effective resistor, and the additional charging power required to make up the resistive dissipation can be very costly. Hence, the low resistance design is preferred.

\section{DESIGN ISSUES}

\section{A. Resistance and Skin Effect}

The load and transmission line resistance varies with frequency due to skin effect. For any given material, the skin depth $\delta_{s}$ is inverse proportional to the square root of the frequency $f$, and the effective resistance $R_{\text {eff }}$ is proportional to the oscillating frequency. Here $\delta_{s}=1 / \sqrt{\pi f \mu_{R} \mu_{0} \sigma}$, and $R_{e f f}=1 / \sigma b \delta_{s}$, where $/$ is the conductor length and $b$ is the conductor width. The material's conductivity, relative permeability, and the free space permeability are described by $\sigma, \mu_{\mathrm{R}}$, and $\mu_{\mathrm{v}}$, respectively.

One can see that lower frequency leads to lower effective resistance. The other factors associated with effective resistance are the length, width, and permeability and material conductivity. For non-magnetic material, the relative permeability is close to unit. The switching device on-state resistance and hardware connection joints resistance also contribute to the total resistance.

In summary, the lower effective resistance can be achieved by using lower frequency, higher skin depth, wider conductor width, shorter conductor length, higher conductivity material, lower switch on-state resistance, and lower connection joint resistance.

\section{B. Inductance Issue}

The total inductance includes the horn inductance, transmission line inductance, series inductance of the capacitor, switch inductance, and circuit loop stray inductance. The external inductance depends on the inductor geometry and material permeability. The internal inductance has frequency dependence.

The voltage across the inductor is $F_{L}(t)=L d I(t) / d t$. Hence, the larger inductor and faster current rate of change requires higher voltage.

The current going through the inductor is the inductively stored energy. For a lossless circuit, the total inductive energy shall be equal to the total capacitive energy storage, i.e. $(1 / 2) \mathrm{LI}^{2}=(1 / 2) \mathrm{CV}^{2}$.

Therefore, we have that the higher the inductance and current, the higher the capacitance and its initial voltage. For a reasonable design, the total inductance shall be kept as low as possible, and the current rise time shall be chosen to accommodate the device operating voltage.

\section{Modularization vs. multi-cell}

Looking into existing horn systems, the commonly used design is multi-cell, multi-switch. The large storage capacitance and very high stored energy make it necessary to divide the capacitors into individual cells and isolate them from each other to avoid catastrophic failure. The very high current switching has to be accomplished by using multiple switches in parallel. The low inductance and low resistance transmission line has to be made by multiple coaxial cables or planar transmission lines. The bigh voltage power supply is often multiple units in parallel sharing common control and combined as a single power source. Large chokes and high voltage diodes are used to isolate multiple storage cells on the charging circuit. At the output side, the multi-cells joint together before transmission line.

The existing multi-cell structure can be extended to a modularized system. Each module has an independent 
power supply, a capacitor storage unit, a main switch of one or two switching devices, and a transmission line. The multiple modules joint together at the horn input. This topology shall provide a better output inductive isolation, higher operability and maintainability, and more flexible charging system. The cost of control and regulation units for multiple power supplies is expected to be eased with the industrial development of multi-chamel digital control systems. The command and status control are usually PLC based system. It has much higher capacity then we normally use and shall be able to serve multimodule system with no significant cost increase.

\section{Major Components}

The major components of a typical horn power supply system include charging power supply, capacitor bank. discharge switch, reverse diode, and transmission line.

In new and proposed designs, self-healing capacitors are being used for the fault tolerance, and increased reliability. This type of capacitor is usually rated under a few kilo-volts. Multiple capacitor cells have to be used to divide capacitor bank into smaller units with lower stored energy per cell for safety concerns.

The discharge switch in favor is the light triggered SCR [9]. The newest in this category is the EUPEC T2563N80. The advantage of this device is its high voltage rating of $8000 \mathrm{~V}$, high forward current rating of $5600 \mathrm{~A} \mathrm{rms}$, and $63 \mathrm{kA}$ surge current. This light triggered gate structure eliminates the high voltage isolation trigger transformers normally used in conventional SCRs, and improves the high voltage hold-off and the noise immumity. Similar devices from $\mathrm{ABB}$ are also being considered.

The trend of new designs is to use solid-state switch, which has much longer lifetime compared to gas discharge switches. The very high current capability required in this system limits the selection to thyristor types for its high power rating per single unit and cost effectiveness. The traditionally used ignitron is mercury vapor filled device. With rapid advancement of solid-state devices, it is being replaced by SCRs. Other solid-state devices, such as IGBT and MOSFET are limited by their current capacity.

Both planar and coaxial transmission lines are being considered. Planar transmission line has the advantage of ultra low resistance. Nevertheless, the open structure of the planar line is more sensitive to the condensation and ionized air at horn. Brookhaven developed rigid coaxial cables is better for higher voltage holding. Robotic arms will be used to remotely connect and disconnect transmission lines from the horn. The mechanical structure of the flexible joint of the transmission line will be a major concern.

\section{DESIGN SIMULATIONS}

The present plan for AGS Super Neutrino Beam Facility is to use two horns at approximately 8 meters apart. The power supply will be house in a service building $50 \mathrm{ft}$ away from the target/horn area. For the 300 -series aluminum being considered in the horn mechanical design, the material skin depth is $3.574 \mathrm{~mm}$ at $833 \mathrm{~Hz}$. The parameters used in following design examples are rough estimates based on similar systems, as listed on Table 2 [4] [5] [6] [7] [8].

Table 2. Similar existing and proposed horn system parameters.

\begin{tabular}{|c|c|c|c|c|c|c|}
\hline Ip & $V_{p}$ & $\operatorname{Tr}$ & C & $\mathrm{L}$ & $\bar{R}$ & Rep. Rate \\
\hline $\mathrm{k} \Lambda$ & $\mathrm{kV}$ & $\mu \mathrm{s}$ & $\mathrm{mF}$ & ${ }_{n} \mathrm{H}$ & $\mu \Omega$ & $\mathrm{Hz}$ \\
\hline \multicolumn{7}{|c|}{ Old AGS Narrow-band } \\
\hline 240 & 12.44 & 58 & 0.850 & 1664 & 9600 & 0.71 \\
\hline \multicolumn{7}{|c|}{ Old AGS Wide-band } \\
\hline 285 & 10.98 & 58 & 1.116 & 1198 & 7500 & 0.71 \\
\hline \multicolumn{7}{|c|}{ FERMI Mini Boone } \\
\hline 170 & 5.35 & 143 & 1.500 & 1340 & 1000 & 5.0 \\
\hline \multicolumn{7}{|c|}{ FERMI NuMi } \\
\hline 205 & 0.97 & 2600 & 900.000 & 2407 & 1031 & 0.53 \\
\hline \multicolumn{7}{|c|}{ CERN } \\
\hline 300 & 7.275 & 81 & 1.075 & 620 & 328 & 75.0 \\
\hline
\end{tabular}

Where, Ip is the pulse current; Vp is the capacitor voltage; $\mathrm{Tr}$ is the pulse rise time; $\mathrm{C}$ is the storage capacitance; $\mathrm{L}$ is the total output circuit inductance of horn, transmission line, and stray; $R$ is the total resistance of horn, transmission line, connection, etc.

\section{A. Basic design}

Let us consider a basic system, as shown in figure 2 , with overall inductance of $2.5 \mu \mathrm{H}$, a horn and line resistance of $2 \mathrm{~m} \Omega$, and a capacitor bank of $16 \mathrm{mF}$. If the initial capacitor voltage is 4000 Volts, the peak output current amplitude is above $280 \mathrm{kA}$ with $314 \mu \mathrm{s}$ rise time.



Figure 2. Basic horn system circuit.

In this design, the diode is used to protect capacitor from excessive reverse voltage. Since the switch is at on state during conversion, the current will continue flow through the output circuit and the stored energy is fully dissipate in each operating cycle. The resistor R2 is chosen to critically damp the current. The capacitor voltage waveform and the horn current waveform simulations are shown in figure 3 .

The stored energy in the capacitor is $128 \mathrm{~kJ}$, and the peak output power is about one Giga-VA. For $2.5 \mathrm{~Hz}$ pulse repetition rate, the minimum charging power supply is $320 \mathrm{~kW}$. The resistance used in the example is tight for 
the chosen frequency. If higher resistance has to be used, then the voltage and capacitance have to be increased accordingly. The figure 4 shows resistance effect of the pulse current under the same capacitance and initial capacitor voltage conditions.
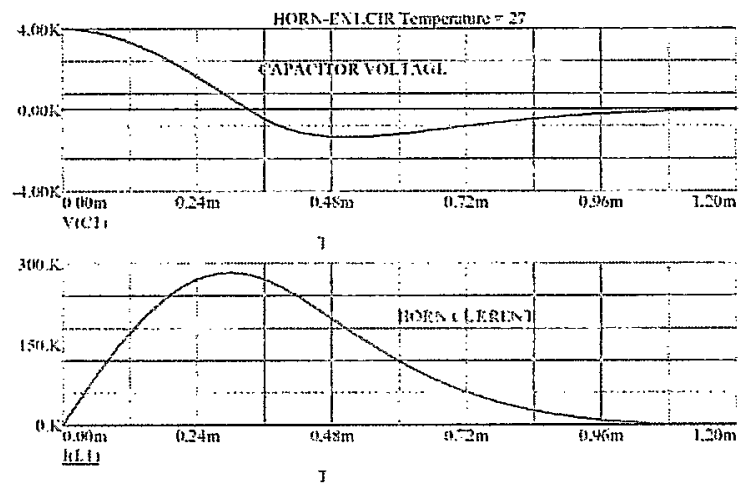

Figure 3. Capacitor voltage and horn current waveform simulations.

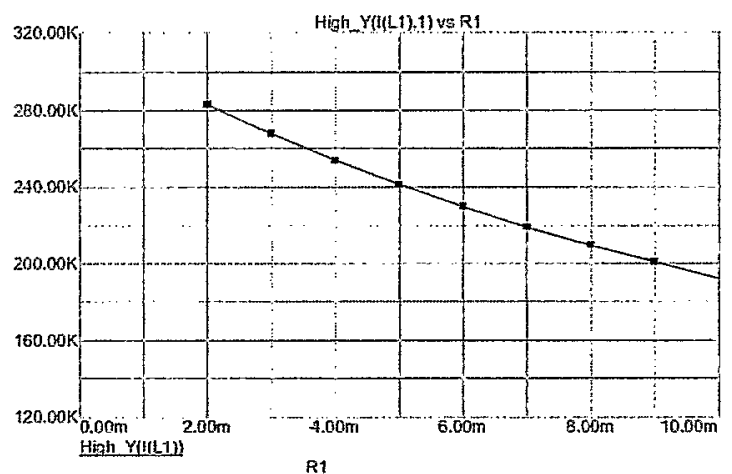

Figure 4. Resistance effect: Horn current amplitude vs. $\mathrm{R} 1$ resistance value.

\section{$E$. Circuit with energy recovery}

To minimize the resistive heat dissipation on horn, two factors may be considered. One is to reduce the horn resistance by mechanical design; another is to recover the electrical energy, as shown in figures 5 and 6 .

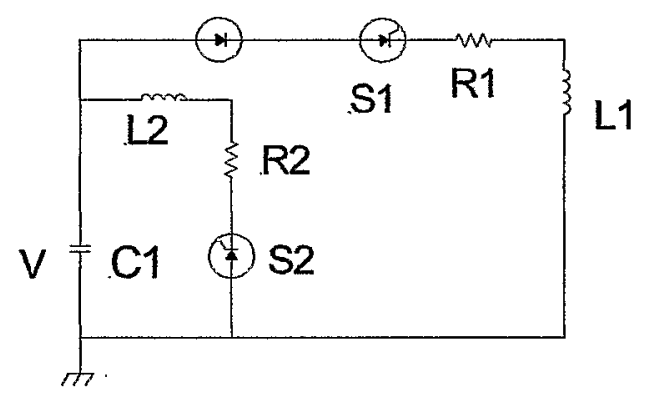

Figure 5. Horn system with energy recovery circuit.
In this particular example, the energy recovered is about $50 \%$. However, additional active components will make the circuit more complicate and the reverse voltage on the capacitor is higher.

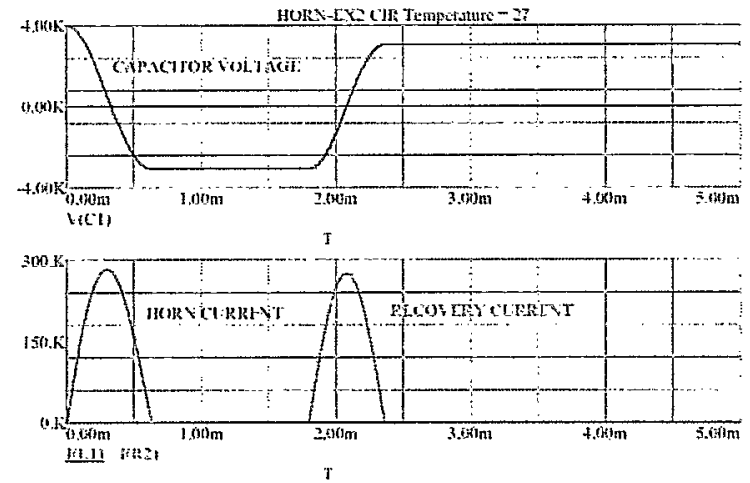

Figure 6. Horn system with recovery circuit: Capacitor voltage, horn current, and recovery current waveform simulations.

The design options of high voltage, high current pulsed system are often limited by the industry development and available components. In this case, the preferred operating voltage is less than $5 \mathrm{kV}$. The total resistance shall be kept to less than or around $2 \mathrm{~m} \Omega$.

\section{ACKNOWLEDGEMENT}

Authors would like to thank Mr. Ken Bourkland of FERMI Laboratory for his advice and useful discussions.

\section{REFERENCES}

[1]. M. Diwan, et al., "AGS Super Neutrino Beam Facility Accelerator and Target System Design", BNL Report \#BNL -71228, April 2003.

[2]. M. Diwan, T. Kirk, W.T. Weng, "Super Neutrino Beam (proton Driver)", internal, Feb. 7, 2003.

[3]. W.T. Weng, M.V. Diwan, "Neutrino Beam Facilities and Projects", PAC2003.

[4]. G. Acquistapace, J.M. Maugain, F. Voelker, "Considerations on a $75 \mathrm{~Hz}$ pulsed horn", CERN, 05, 2000.

[5]. K. Bourkland, C. Jensen, D. Tinsley, "High Current Pulse Striplines", 2001 PPPS.

[6]. K. Bourkland, K. Roon, D. Tinsley, "205 kA Pulse Power Supply for Neutrino Focusing Horn", 2002 PMS.

[7]. G. Grawer, "Tnvestigation of the possibility to build a $400 \mathrm{kA}$ pulse current generator to drive a magnetic horn", CERN-NUFACT Note 038, July $19,2000$.

[8]. J. Sandberg, et.al. "The Neutrino Horn 300 Kiloampere Pulsed Power Supply at Brookhaven National Laboratory", PAC 1987.

[9]. K. Bourkland, private communication. 